\title{
Effect of Mineral Admixture, W/B Ratio and Elevated Temperature on Strength of Lightweight Expanded Clay Aggregate Concrete
}

\author{
Veeramala sravan ${ }^{1}$, Tangudu Manoj ${ }^{2}$ and Dr. M. V. Seshagiri Rao ${ }^{3}$ \\ ${ }^{1}$ PG Scholar, CVR College of Engineering/Civil Engg. Department, Hyderabad, India \\ Email: sravanveeramalla999@gmail.com \\ ${ }^{2}$ Asst. Professor, CVR College of Engineering/Civil Engg. Department, Hyderabad, India \\ Email: manoj.tangudu03@gmail.com \\ ${ }^{3}$ Professor, CVR College of Engineering/Civil Engg. Department, Hyderabad, India \\ Email: rao_vs_meduri@yahoo.com
}

\begin{abstract}
The present paper focused on development of structural lightweight concrete by using light weight expanded clay aggregate (LECA) with unit weight of $1700 \mathrm{~kg} / \mathrm{m}^{3}$ to 1900 $\mathrm{kg} / \mathrm{m}^{3}$ and compressive strength of $40 \mathrm{MPa}$. In this study, investigation was done using LECA as coarse aggregate in concrete by replacing normal aggregate in three differing volume fractions i.e., $30 \%, 40 \%$ and $50 \%$ with different $w / b$ ratios $0.40,0.50$ and 0.60 to produce lightweight expanded clay aggregate concrete (LECAC) with addition of chemical admixture. For that, concrete mix design was done using IS method and replacement of lightweight aggregate was calculated based on volume batching. The effect of mineral admixture (i.e., $10 \%$ microsilica) on the properties of lightweight expanded clay aggregate concrete and normal weight aggregate concrete such as workability of fresh concrete, compressive strength and flexural strength of concrete were compared. The studies also include the effect of elevated temperature on light weight expanded clay aggregate concrete and normal weight aggregate concrete.
\end{abstract}

Index Terms: Normal weight aggregate concrete (NWAC), Light weight expanded clay aggregate (LECA). Light weight expanded clay aggregate concrete (LECAC), Elevated temperature.

\section{INTRODUCTION}

Light weight expanded clay aggregate is a special type of aggregate which is formed by heating clay with no or very little content of lime. The clay is dried, heated and burned in rotary kiln at a temperature of $1100^{\circ} \mathrm{C}-1300^{\circ} \mathrm{C}$. Since it is exposed to high temperature as a result, gas is released inside the pellets and entrapped in it during cooling whilst the organic compounds burnt off forcing the pellets to bloated producing ceramic pellets with porous and formed a honeycombed structure. Outer surface of each granule is sintered. Pore structure gives light weight and high crushing resistance, thermal as well as sound insulation to the material. It has a circular or a potato shape formed due to circular movements in the kiln.

LECA consist of rounded pellets with a vesicular texture. In generally, LECA is a dark brown or reddish or grey in colour. These differences in colours could be associated to the varieties in LECA chemical composition. LECA is available in different sizes varying $0.1 \mathrm{~mm}$ to $25 \mathrm{~mm}$, which is suitable for fine aggregate, coarse aggregate, and both of them. Bulk density of LECA ranges from 250 to $750 \mathrm{~kg} / \mathrm{m}^{3}$. It is used in making lightweight concrete products and for other uses.

The aim of the present study is to determine the mechanical properties of LECAC. The LECA was used in place of normal weight coarse aggregate in three differing volume fractions i.e., LECAC $30(30 \%$ LECA $+70 \%$ GRAVEL), LECAC40(40\% LECA +60\% GRAVEL) and LECAC50 (50\% LECA+ 50\% GRAVEL), with different $\mathrm{w} / \mathrm{b}$ ratios $0.40,0.50$ and 0.60 to produce a lightweight expanded clay aggregate concrete (LECAC). The effect of elevated temperature on the mechanical properties of the light weight expanded clay aggregate concrete is also studied.

\section{LITERATURE REVIEW}

Alaa M.Rashad studied use of light weight expanded clay aggregate as a building material. An overview of LECA as a building material is given. According to the study conclusions are LECA has a positive effect on workability, decreases the specific tensile creep. It improves sound isolation.

K. Akcaozoglu, S.Akcaozoglu made experimentally studied on the effect of elevated temperature on the lightweight concrete produced by expanded clay aggregate and calcium aluminate cement. The residual strength of mixtures produced by expanded clay aggregate were higher than the concrete produced by natural aggregates.

Abhijitsinh Parmar, Urvish Patel studied the fresh concrete properties using Lightweight Expanded clay aggregate (LECA) and Expanded Polystyrene Beads (EPS) by replacing coarse aggregates. LECA can be used as a replacement for coarse aggregate compared to EPS with low workability.

Anil Kumar R, P. Prakash studied the mechanical properties of structural concrete by blending cinder and LECA. The strength properties of lightweight concrete produced by replacing coarse aggregate for M30 grade of concrete were studied. Concrete with $60 \%$ Cinder and LECA $40 \%$ had an average compressive strength of $36.52 \mathrm{~N} / \mathrm{mm}^{2}$ and split tensile strength of $2.5 \mathrm{~N} / \mathrm{mm}^{2}$. 
Omar A. Abdulkareem, A.M. Mustafa al Bakri examined that effect of elevated temperatures on the thermal behavior and mechanical performance of fly ash geopolymer paste, mortar and light weight concrete. They presented the characteristics of the FA geopolymer paste, mortar and LWAGC before and after exposed to elevated temperatures of $400^{\circ} \mathrm{C}, 600^{\circ} \mathrm{C}$ and $800^{\circ} \mathrm{C}$.

\section{Materials}

Cement

Ordinary Portland cement of 53 grade cement is used. The Physical properties are tested according to IS 40311998 and results are tabulated in Table I.

$$
\text { TABLE I. }
$$

PHYSICAL PROPERTIES OF OPC 53

\begin{tabular}{|c|l|l|l|l|}
\hline $\begin{array}{c}\mathrm{S} \\
\mathrm{N} \\
\mathrm{o}\end{array}$ & Property & $\begin{array}{l}\text { Test } \\
\text { method }\end{array}$ & $\begin{array}{l}\text { Test } \\
\text { Results }\end{array}$ & $\begin{array}{l}\text { IS } \\
\text { Specification }\end{array}$ \\
\hline 1 & Fineness & Sieve test & $2.4 \%$ & $10 \%$ \\
\hline 2 & $\begin{array}{l}\text { Specific } \\
\text { Gravity }\end{array}$ & $\begin{array}{l}\text { Specific } \\
\text { gravity } \\
\text { bottle }\end{array}$ & 3.14 & $3.10-3.25$ \\
\hline 3 & $\begin{array}{l}\text { Normal } \\
\text { Consistenc } \\
\text { y }\end{array}$ & $\begin{array}{l}\text { Vicat } \\
\text { apparatus }\end{array}$ & $31 \%$ & $26-33 \%$ \\
\hline 4 & $\begin{array}{l}\text { Initial } \\
\text { Setting } \\
\text { time }\end{array}$ & $\begin{array}{l}\text { Vicat } \\
\text { apparatus }\end{array}$ & 45 min & $\begin{array}{l}\text { Not less } \\
\text { than 30 min }\end{array}$ \\
\hline
\end{tabular}

TABLE III

QUANTITIES REQUIRED FOR $1 \mathrm{M}^{3}$

\begin{tabular}{|c|c|c|c|c|c|c|c|c|}
\hline \multirow[b]{2}{*}{$\begin{array}{l}\mathrm{w} / \mathrm{c} \\
\text { ratio }\end{array}$} & \multirow[b]{2}{*}{$\begin{array}{l}\text { Cement } \\
\mathrm{Kg} / \mathrm{m}^{3}\end{array}$} & \multirow[b]{2}{*}{$\begin{array}{c}\text { Micro } \\
\text { silica } \\
\mathrm{Kg} / \mathrm{m}^{3}\end{array}$} & \multirow[b]{2}{*}{$\begin{array}{l}\text { Water } \\
\mathrm{Kg} / \mathrm{m}^{3}\end{array}$} & \multirow[b]{2}{*}{$\begin{array}{c}\text { SP dosage } \\
\%\end{array}$} & \multirow[b]{2}{*}{$\begin{array}{c}\text { Fine } \\
\text { aggregate } \\
\mathrm{Kg} / \mathrm{m}^{3}\end{array}$} & \multicolumn{3}{|c|}{ Coarse aggregate $\mathrm{Kg} / \mathrm{m}^{3}$} \\
\hline & & & & & & $\begin{array}{c}\text { LECAC30 } \\
\text { (30\%LECA } \\
+70 \% \text { Gravel) } \\
\mathrm{Kg} / \mathrm{m}^{3} \\
\end{array}$ & $\begin{array}{c}\text { LECAC40 } \\
(40 \% \text { LECA } \\
+60 \% \text { Gravel }) \\
\mathrm{Kg} / \mathrm{m}^{3} \\
\end{array}$ & $\begin{array}{c}\text { LECAC50 } \\
(50 \% \text { LECA } \\
+50 \% \text { Gravel }) \\
\mathrm{Kg} / \mathrm{m}^{3} \\
\end{array}$ \\
\hline 0.4 & 331 & 0 & 132 & $1.5 \%$ & 711 & $96.228+952.56$ & $128.30+816.48$ & $160.38+680.4$ \\
\hline 0.4 & 331 & 33.1 & 145 & $1.5 \%$ & 702 & $95.04+940.8$ & $126.72+806.04$ & $158.4+672$ \\
\hline 0.5 & 331 & 0 & 165 & $1 \%$ & 718 & $89.1+882$ & $118.8+756$ & $148.5+630$ \\
\hline 0.5 & 331 & 33.1 & 182 & $1 \%$ & 704 & $84.38+860$ & $108.504+740.88$ & $135.64+575.4$ \\
\hline 0.6 & 331 & 0 & 199 & $0 \%$ & 723 & $82.368+815.36$ & $109.824+698.88$ & $137.2+582.4$ \\
\hline 0.6 & 331 & 33.1 & 218 & $0 \%$ & 697 & $80.388+795.76$ & $107.18+682.08$ & $133.98+568.4$ \\
\hline
\end{tabular}

\section{FINE AGGREGATE}

River sand is used as fine aggregate. The requirements of sand is confirming to IS: $383-1970$ and the properties of fine aggregate tested are tabulated in Table II.

\section{COARSE AGGREGATE}

Gravel

Aggregates passing from $12 \mathrm{~mm}$ and retained on $20 \mathrm{~mm}$ aggregates are used. Properties of coarse aggregates are tabulated in Table II.

LECA

Light weight expanded clay aggregate of size from $8 \mathrm{~mm}$ to $12 \mathrm{~mm}$ is used as coarse aggregate. properties of LECA are tested and results are tabulated in Table II.

TABLE II.

Physical Properties of F.A, C.A(GRAVEl ANd LeCA)

\begin{tabular}{|c|c|c|c|c|}
\hline \multirow{2}{*}{ S.No. } & Property & \multirow{2}{*}{$\begin{array}{c}\text { Fine } \\
\text { Aggregate }\end{array}$} & \multicolumn{2}{|c|}{ Coarse Aggregate } \\
\cline { 4 - 5 } & & Gravel & LECA \\
\hline 1 & $\begin{array}{c}\text { Fineness } \\
\text { modulus }\end{array}$ & 3.08 & 6.41 & 5.84 \\
\hline 2 & $\begin{array}{c}\text { Specific } \\
\text { gravity }\end{array}$ & 2.6 & 2.88 & 0.66 \\
\hline 3 & Bulk density & $\begin{array}{c}1440 \\
\mathrm{Kg} / \mathrm{m}^{3}\end{array}$ & $\begin{array}{c}1450 \\
\mathrm{~kg} / \mathrm{m}^{3}\end{array}$ & $\begin{array}{c}413 \\
\mathrm{~kg} / \mathrm{m}^{3}\end{array}$ \\
\hline 4 & $\begin{array}{c}\text { Water } \\
\text { absorption }\end{array}$ & $1 \%$ & $0.6 \%$ & $15 \%$ \\
\hline
\end{tabular}

Water

Portable water is used in the preparation of concrete mixes. Super Plasticizer

CONPLAST SP 430 is used as the chemical admixture.

Micro Silica

Micro silica is used as a mineral admixture in the concrete. 


\section{EXPERIMENTAL STUDY}

Workability

The workability is measured by conducting the Slump cone test using standard Procedure prescribed in IS: 1199 - 1959. Results are tabulated in Table IV.

TABLE IV.

WORKABILITY AND UNIT WEIGHT

\begin{tabular}{|c|c|c|c|c|c|c|}
\hline \multirow{2}{*}{$\begin{array}{l}\mathrm{w} / \mathrm{c} \\
\text { ratio. }\end{array}$} & \multirow{2}{*}{$\begin{array}{c}\text { LECA } \\
\text { vol. } \\
\text { fraction } \\
\%\end{array}$} & \multirow{2}{*}{$\begin{array}{c}\mathrm{SP} \\
\text { dosage }\end{array}$} & \multicolumn{2}{|c|}{$\begin{array}{l}\text { Without Micro } \\
\text { silica }\end{array}$} & \multicolumn{2}{|c|}{$\begin{array}{l}\text { With micro } \\
\text { silica }\end{array}$} \\
\hline & & & $\begin{array}{l}\text { Slump } \\
(\mathrm{mm})\end{array}$ & $\begin{array}{c}\text { Unit } \\
\text { weight } \\
\left(\mathrm{kg} / \mathrm{m}^{3}\right)\end{array}$ & $\begin{array}{c}\text { Slump } \\
(\mathrm{mm})\end{array}$ & $\begin{array}{c}\text { Unit } \\
\text { weight } \\
\left(\mathrm{kg} / \mathrm{m}^{3}\right)\end{array}$ \\
\hline \multirow{4}{*}{0.40} & 0 & $1.5 \%$ & 50 & 2288 & 47 & 2388 \\
\hline & 30 & $1.5 \%$ & 52 & 1829.8 & 50 & 1890.2 \\
\hline & 40 & $1.5 \%$ & 52 & 1754.2 & 54 & 1825.1 \\
\hline & 50 & $1.5 \%$ & 55 & 1731.2 & 58 & 1746.2 \\
\hline \multirow{4}{*}{0.50} & 0 & $1 \%$ & 52 & 2314 & 49 & 2341 \\
\hline & 30 & $1 \%$ & 56 & 1821.4 & 52 & 1882.3 \\
\hline & 40 & $1 \%$ & 60 & 1749.5 & 58 & 1820.4 \\
\hline & 50 & $1 \%$ & 67 & 1728.6 & 66 & 1745.5 \\
\hline \multirow{4}{*}{0.60} & 0 & $0 \%$ & 64 & 2256 & 62 & 2286 \\
\hline & 30 & $0 \%$ & 70 & 1809 & 66 & 1874.4 \\
\hline & 40 & $0 \%$ & 73 & 1747.8 & 68 & 1813.5 \\
\hline & 50 & $0 \%$ & 88 & 1722.5 & 75 & 1742.9 \\
\hline
\end{tabular}

\section{Compressive strength}

Cube size of $150 \mathrm{~mm} \times 150 \mathrm{~mm} \times 150 \mathrm{~mm}$ cube specimens are caste and cured for 28 days and tested under Automatic compression testing machine of $3000 \mathrm{KN}$ capacity and the results are tabulated in Table $\mathrm{V}$.
TABLE V.

PERCENTAGE INCREASE IN COMPRESSIVE STRENGTH ATAGE OF 28 DAYS WITH AND WITHOUT ADDITION OF MINERAL ADMIXTURE (MICROSILICA).

\begin{tabular}{|c|c|c|c|c|}
\hline \multirow{2}{*}{$\begin{array}{l}\mathrm{w} / \mathrm{c} \\
\text { rat } \\
\text { io }\end{array}$} & \multirow{2}{*}{$\begin{array}{c}\text { leca } \\
\text { vol. } \\
\text { fraction } \\
\%\end{array}$} & \multicolumn{2}{|c|}{$\begin{array}{l}\text { compressive strength } \\
\text { mpa }\end{array}$} & \multirow{2}{*}{$\begin{array}{c}\% \text { of increase in } \\
\text { compressive } \\
\text { strength }\end{array}$} \\
\hline & & $\begin{array}{l}\text { without } \\
\text { micro } \\
\text { silica }\end{array}$ & $\begin{array}{l}\text { with } \\
\text { micro } \\
\text { silica }\end{array}$ & \\
\hline \multirow{4}{*}{0.4} & 0 & 55 & 59 & 7.2 \\
\hline & 30 & 27.04 & 29.2 & 7.9 \\
\hline & 40 & 24.93 & 27.04 & 8.5 \\
\hline & 50 & 20.56 & 24.9 & 21.1 \\
\hline \multirow{4}{*}{0.5} & 0 & 52 & 56.36 & 8.4 \\
\hline & 30 & 24.7 & 27.04 & 9.5 \\
\hline & 40 & 23.3 & 25.73 & 10.4 \\
\hline & 50 & 18.8 & 22.66 & 20.53 \\
\hline \multirow{4}{*}{0.6} & 0 & 46.6 & 50.7 & 9.2 \\
\hline & 30 & 22.1 & 24.24 & 9.7 \\
\hline & 40 & 20.66 & 22.88 & 10.7 \\
\hline & 50 & 18.2 & 21.53 & 18.34 \\
\hline
\end{tabular}

Flexural strength

Prisms of size $100 \mathrm{~mm} \times 100 \mathrm{~mm} \times 500 \mathrm{~mm}$ were caste and cured for 28 days and tested in flexural testing machine and results are tabulated in Table VI and Table VII.

TABLE VI

FLEXURAL STRENGTH OF NWAC MIXES WiTH AND WITHOUT MICROSILICA

\begin{tabular}{|c|c|c|}
\hline \multirow{2}{*}{ W/C ratio } & \multicolumn{2}{|c|}{ Flexural strength $(\mathrm{MPa})$} \\
\cline { 2 - 3 } & $\begin{array}{c}\text { Without Micro } \\
\text { silica }\end{array}$ & With Micro silica \\
\hline 0.4 & 5.19 & 5.3 \\
\hline 0.5 & 4.9 & 5.2 \\
\hline 0.6 & 4.7 & 4.9 \\
\hline
\end{tabular}

TABLE VII.

FLeXURAL STRENGTH OF LECAC30 MiXes With AND Without Micro SILICA

\begin{tabular}{|c|c|c|}
\hline \multirow{2}{*}{ W/C ratio } & \multicolumn{2}{|c|}{ Flexural strength (MPa) } \\
\cline { 2 - 3 } & Without Micro silica & $\begin{array}{c}\text { With Micro } \\
\text { silica }\end{array}$ \\
\hline 0.4 & 3.64 & 3.8 \\
\hline 0.5 & 3.4 & 3.6 \\
\hline 0.6 & 3.2 & 3.4 \\
\hline
\end{tabular}


Effect of elevated temperature

High temperature furnace of $1000^{\circ} \mathrm{C}$ capacity is used to evaluate the fire resistance. The casted cubes are placed in the high temperature furnace for $02 \mathrm{hrs}$ at different temperatures $200^{\circ} \mathrm{C}, 400^{\circ} \mathrm{C}, 600^{\circ} \mathrm{C} \& 800^{\circ} \mathrm{C}$. After two hours cubes were taken out and tested residual compressive strength, weight loss and the results are tabulated in Table VIII - Table XIII.

TABLE VIII.

ResiduAL COMPRESSIVE STRENGTH OF NWAC CUBES

\begin{tabular}{|c|c|c|c|c|c|}
\hline \multirow[b]{2}{*}{$\begin{array}{l}\text { W/ } \\
\mathrm{C}\end{array}$} & \multirow[b]{2}{*}{$\begin{array}{l}\text { Te } \\
\text { mp } \\
\text { erat } \\
\text { ure } \\
\left({ }^{\circ} \mathrm{C}\right)\end{array}$} & \multicolumn{2}{|c|}{$\begin{array}{c}\text { Compressive } \\
\text { strength (MPa) }\end{array}$} & \multicolumn{2}{|c|}{$\%$ of variation } \\
\hline & & $\begin{array}{l}\text { NWAC } \\
\text { Without } \\
\text { micro } \\
\text { silica }\end{array}$ & $\begin{array}{l}\text { NWAC } \\
\text { with } \\
\text { micro } \\
\text { silica }\end{array}$ & $\begin{array}{c}\text { NWAC } \\
\text { without } \\
\text { micro } \\
\text { silica }\end{array}$ & $\begin{array}{l}\text { NWAC } \\
\text { with } \\
\text { micro } \\
\text { silica } \\
\end{array}$ \\
\hline \multirow{5}{*}{0.4} & 0 & 55 & 59 & 0 & 0 \\
\hline & 200 & 50.63 & 52.4 & 7.9 & 11.1 \\
\hline & 400 & 49.2 & 49.4 & 10.5 & 16.2 \\
\hline & 600 & 44.9 & 45 & 18.3 & 23.7 \\
\hline & 800 & 18 & 21 & 67.2 & 64.4 \\
\hline \multirow{5}{*}{0.5} & 0 & 52 & 57 & 0 & 0 \\
\hline & 200 & 44.8 & 47.2 & 13.8 & 17.1 \\
\hline & 400 & 42.3 & 35.4 & 18.6 & 37.8 \\
\hline & 600 & 38.4 & 28.3 & 26.1 & 50.3 \\
\hline & 800 & 11.65 & 18.4 & 77 & 67.7 \\
\hline \multirow{5}{*}{0.6} & 0 & 46.6 & 50.9 & 0 & 0 \\
\hline & 200 & 36.9 & 38.9 & 20.8 & 23.5 \\
\hline & 400 & 32.16 & 32.63 & 30.9 & 35.8 \\
\hline & 600 & 27.14 & 22.6 & 41.7 & 55 \\
\hline & 800 & 9.4 & 12.8 & 79.8 & 74.8 \\
\hline
\end{tabular}

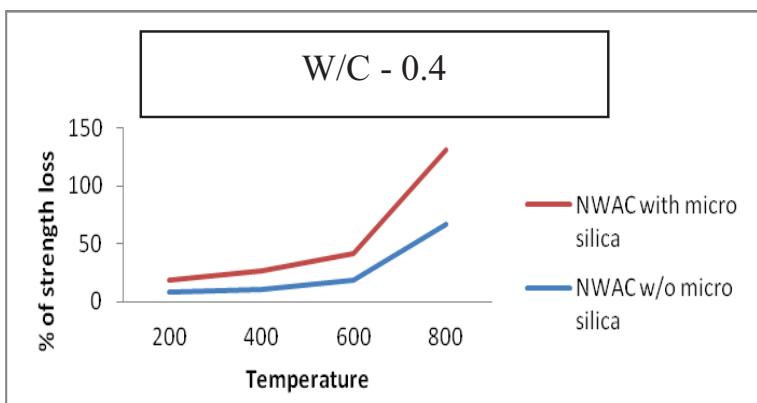

Figure 1. Loss of compressive strength at different temperatures NWAC

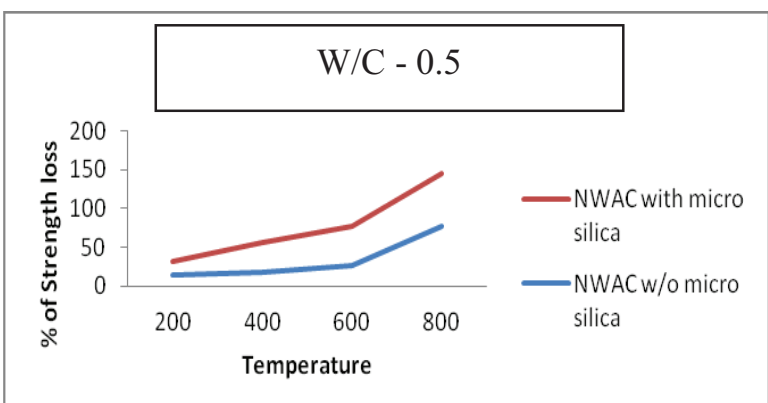

Figure 2. loss of compressive strength at different temperatures NWAC

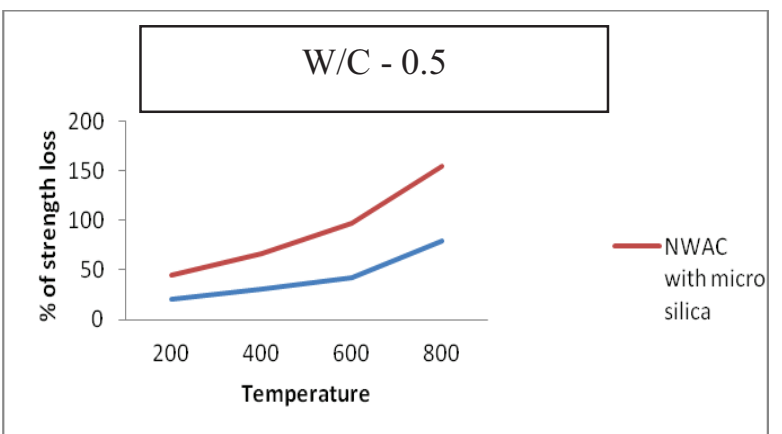

Figure 3. Loss of compressive strength at different temperatures NWAC

TABLE IX.

REsidual COMPRESSIVE STRENGTH OF LECAC30 CUBES

\begin{tabular}{|c|c|c|c|c|c|}
\hline \multirow[t]{2}{*}{$\begin{array}{l}\mathrm{W} \\
/ \mathrm{C}\end{array}$} & \multirow{2}{*}{$\begin{array}{l}\mathrm{Te} \\
\mathrm{mp} \\
\text { erat } \\
\text { ure } \\
\left({ }^{\circ} \mathrm{C}\right)\end{array}$} & \multicolumn{2}{|c|}{$\begin{array}{l}\text { Compressive } \\
\text { strength } \\
(\mathrm{MPa})\end{array}$} & \multicolumn{2}{|c|}{$\%$ of variation } \\
\hline & & $\begin{array}{c}\text { LECAC } \\
30 \\
\text { withou } \\
\mathrm{t} \\
\text { micro } \\
\text { silica }\end{array}$ & $\begin{array}{c}\text { LECAC } \\
30 \\
\text { with } \\
\text { micro } \\
\text { silica }\end{array}$ & $\begin{array}{c}\text { LECAC } \\
30 \\
\text { without } \\
\text { micro } \\
\text { silica }\end{array}$ & $\begin{array}{c}\text { LEC } \\
\text { AC } \\
30 \\
\text { with } \\
\text { micro } \\
\text { silica }\end{array}$ \\
\hline \multirow{5}{*}{0.4} & 0 & 27.04 & 29.20 & 0 & 0 \\
\hline & 200 & 26.6 & 28.2 & 1.6 & 3.4 \\
\hline & 400 & 24.7 & 27.96 & 8.6 & 4.2 \\
\hline & 600 & 22.5 & 26.4 & 16.8 & 9.5 \\
\hline & 800 & 18.53 & 21.3 & 31.4 & 27 \\
\hline \multirow{5}{*}{0.5} & 0 & 24.7 & 27.2 & 0 & 0 \\
\hline & 200 & 23.73 & 27 & 3.9 & 7.3 \\
\hline & 400 & 21.76 & 24.56 & 11.9 & 9.7 \\
\hline & 600 & 18.92 & 21.1 & 23.4 & 22.4 \\
\hline & 800 & 10.93 & 15.1 & 55.7 & 44.4 \\
\hline \multirow{5}{*}{0.6} & 0 & 22.1 & 23.9 & 0 & 0 \\
\hline & 200 & 18.25 & 21.6 & 17.4 & 19.6 \\
\hline & 400 & 17.86 & 21.5 & 19.1 & 10.0 \\
\hline & 600 & 16.5 & 18.1 & 25.3 & 24.2 \\
\hline & 800 & 7.54 & 12 & 66 & 49.7 \\
\hline
\end{tabular}

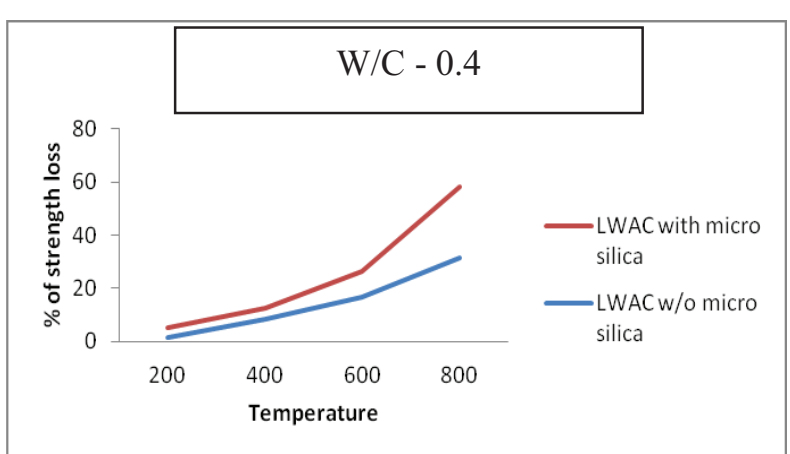

Figure 4. Loss of compressive strength at different temperatures LECAC30 


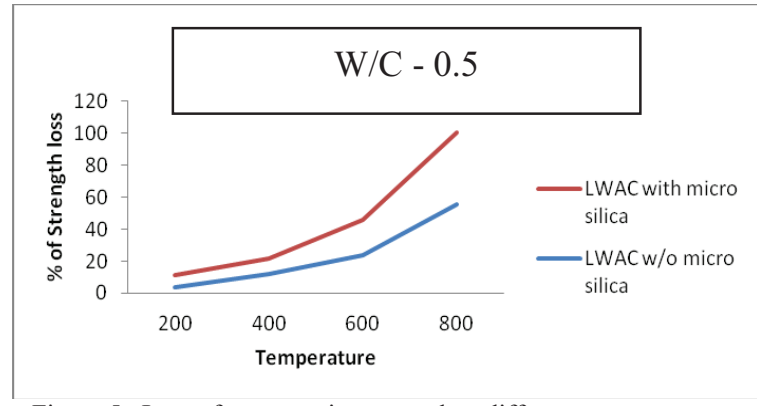

Figure 5. Loss of compressive strength at different temperatures LECAC 30

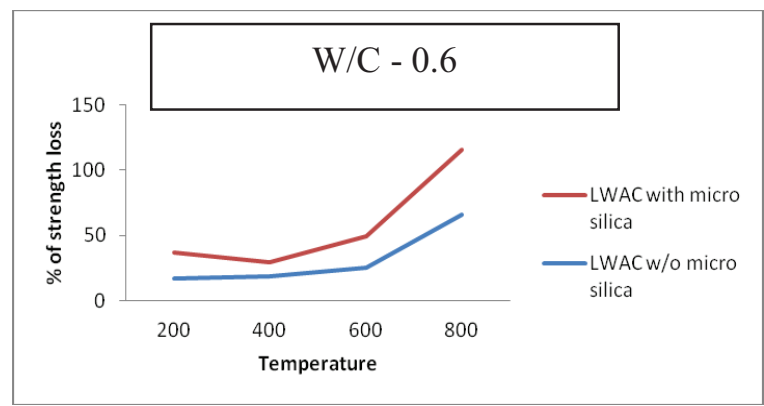

Figure 6 . Loss of compressive strength at different temperatures -

$$
\text { LECAC30 }
$$

TABLE X

Average Percentage of Weight Loss of Nwac Without MICROSILICA AT DIFFERENT TEMPERATURES

\begin{tabular}{|c|c|c|c|c|}
\hline $\mathrm{W} / \mathrm{C}$ & $\begin{array}{c}\text { Temperature } \\
\left({ }^{\circ} \mathrm{C}\right)\end{array}$ & $\begin{array}{l}\text { Weight } \\
\text { before } \\
\text { placing in } \\
\text { furnace } \\
(\mathrm{kg})\end{array}$ & $\begin{array}{l}\text { Weight } \\
\text { after } \\
\text { placing } \\
\text { in } \\
\text { furnace } \\
(\mathrm{kg})\end{array}$ & $\begin{array}{c}\% \text { of } \\
\text { variati } \\
\text { on }\end{array}$ \\
\hline \multirow{4}{*}{0.4} & 200 & 8.57 & 8.52 & 1.0 \\
\hline & 400 & 8.55 & 8.25 & 3.5 \\
\hline & 600 & 8.37 & 8.02 & 4.1 \\
\hline & 800 & 8.48 & 8.03 & 5.3 \\
\hline \multirow{4}{*}{0.5} & 200 & 8.39 & 8.29 & 1.1 \\
\hline & 400 & 8.58 & 8.28 & 3.4 \\
\hline & 600 & 8.45 & 7.99 & 5.4 \\
\hline & 800 & 8.43 & 7.92 & 6.0 \\
\hline \multirow{4}{*}{0.6} & 200 & 8.54 & 8.4 & 1.6 \\
\hline & 400 & 8.29 & 7.91 & 4.5 \\
\hline & 600 & 8.36 & 7.88 & 5.7 \\
\hline & 800 & 8.29 & 7.58 & 8.5 \\
\hline
\end{tabular}

TABLE XI.

Average Percentage Of Weight Loss Of NWAC With MICROSILICA AT DIFFERENT TEMPERATURES

\begin{tabular}{|c|c|c|c|c|}
\hline $\mathrm{W} / \mathrm{C}$ & $\begin{array}{r}\text { Tempera } \\
\text { ture } \\
\left({ }^{\circ} \mathrm{C}\right)\end{array}$ & $\begin{array}{l}\text { Weight } \\
\text { before } \\
\text { placing } \\
\text { in } \\
\text { furnace } \\
(\mathrm{kg})\end{array}$ & $\begin{array}{l}\text { Weight } \\
\text { after } \\
\text { placing } \\
\text { in } \\
\text { furnace } \\
(\mathrm{kg})\end{array}$ & $\begin{array}{l}\quad \% \text { of } \\
\text { variati } \\
\text { on }\end{array}$ \\
\hline \multirow{4}{*}{0.4} & 200 & 8.32 & 8.23 & 1.6 \\
\hline & 400 & 8.4 & 8.03 & 4.4 \\
\hline & 600 & 8.29 & 7.66 & 7.5 \\
\hline & 800 & 8.37 & 7.65 & 8.6 \\
\hline \multirow{4}{*}{0.5} & 200 & 8.55 & 8.44 & 1.2 \\
\hline & 400 & 8.04 & 7.66 & 4.7 \\
\hline & 600 & 8.16 & 7.59 & 6.9 \\
\hline & 800 & 8.42 & 7.8 & 7.3 \\
\hline \multirow{4}{*}{0.6} & 200 & 8.25 & 8.12 & 1.6 \\
\hline & 400 & 8.36 & 7.9 & 5.5 \\
\hline & 600 & 8.35 & 7.52 & 9.9 \\
\hline & 800 & 8.37 & 7.48 & 10.6 \\
\hline
\end{tabular}

\section{Temperature v/s average percentage of weight loss 0.4 W/C ratio w/o microsilica with micosilica}

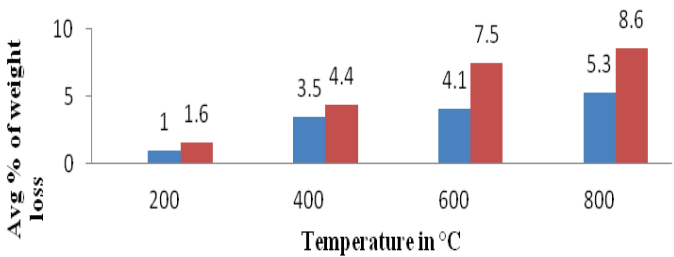

Figure 7. Average percentage loss of weight at different temperaturesNWAC

\section{Temperature v/s average percentage of weight loss}

0.5 W/C ratio w/omicrosilica $\quad$ with micosilica

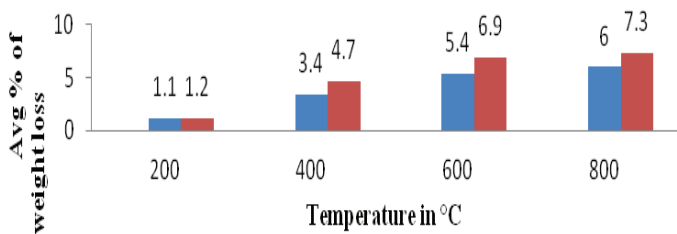

Figure 8 . Average percentage loss of weight at different temperaturesNWAC 


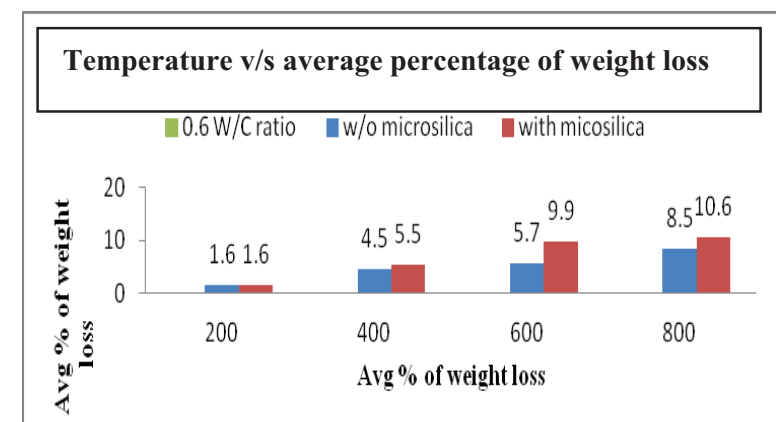

Figure 9. Average percentage loss of weight at different temperaturesNWAC

TABLE XII.

Average Percentage of Weight Loss of Lecac30 Without MicRosilicA AT DiFFERENT TEMPERATURES

\begin{tabular}{|c|c|c|c|c|}
\hline $\mathrm{W} / \mathrm{C}$ & $\begin{array}{c}\text { Temperature } \\
\left({ }^{\circ} \mathrm{C}\right)\end{array}$ & $\begin{array}{l}\text { Weight } \\
\text { before } \\
\text { placing } \\
\text { in } \\
\text { furnace } \\
(\mathrm{kg})\end{array}$ & $\begin{array}{l}\text { Weight } \\
\text { before } \\
\text { placing } \\
\text { in } \\
\text { furnace } \\
(\mathrm{kg})\end{array}$ & $\begin{array}{c}\quad \% \text { of } \\
\text { variation }\end{array}$ \\
\hline \multirow{3}{*}{0.4} & 200 & 7.58 & 7.37 & 2.7 \\
\hline & 400 & 7.48 & 7.09 & 5.2 \\
\hline & 600 & 7.53 & 7.13 & 5.3 \\
\hline \multirow{4}{*}{0.5} & 800 & 7.58 & 7.1 & 6.3 \\
\hline & 200 & 7.26 & 7.16 & 1.3 \\
\hline & 400 & 7.43 & 7.02 & 5.5 \\
\hline & 600 & 7.5 & 7.03 & 6.2 \\
\hline \multirow{4}{*}{0.6} & 800 & 7.5 & 6.88 & 7.2 \\
\hline & 200 & 7.29 & 7.19 & 1.3 \\
\hline & 400 & 7.4 & 7.06 & 4.5 \\
\hline & 600 & 7.3 & 6.72 & 7.9 \\
\hline
\end{tabular}

TABLE XIII.

AVERAGE PERCENTAGE OF WEIGHT LOSS OF LECAC30 WITH MICROSILICA AT DIFFERENT TEMPERATURES

\begin{tabular}{|c|c|c|c|c|}
\hline $\mathrm{W} / \mathrm{C}$ & $\begin{array}{c}\text { Temperature } \\
\left({ }^{\circ} \mathrm{C}\right)\end{array}$ & $\begin{array}{l}\text { Weight } \\
\text { before } \\
\text { placing } \\
\text { in } \\
\text { furnace } \\
\text { (kg) }\end{array}$ & $\begin{array}{l}\text { Weight } \\
\text { after } \\
\text { placing } \\
\text { in } \\
\text { furnace } \\
\text { (kg) }\end{array}$ & $\begin{array}{c}\% \text { of } \\
\text { variation }\end{array}$ \\
\hline \multirow{4}{*}{0.4} & 200 & 7.505 & 7.3 & 2.7 \\
\hline & 400 & 7.57 & 7.22 & 5.6 \\
\hline & 600 & 7.54 & 6.99 & 6.8 \\
\hline & 800 & 7.57 & 6.92 & 8.5 \\
\hline \multirow{4}{*}{0.5} & 200 & 7.58 & 7.45 & 1.7 \\
\hline & 400 & 7.4 & 7.13 & 3.6 \\
\hline & 600 & 7.64 & 7.11 & 6.9 \\
\hline & 800 & 7.44 & 6.83 & 8.5 \\
\hline \multirow{4}{*}{0.6} & 200 & 7.48 & 7.34 & 1.8 \\
\hline & 400 & 7.25 & 6.79 & 6.3 \\
\hline & 600 & 7.46 & 6.78 & 9.1 \\
\hline & 800 & 7.42 & 6.71 & 9.5 \\
\hline
\end{tabular}

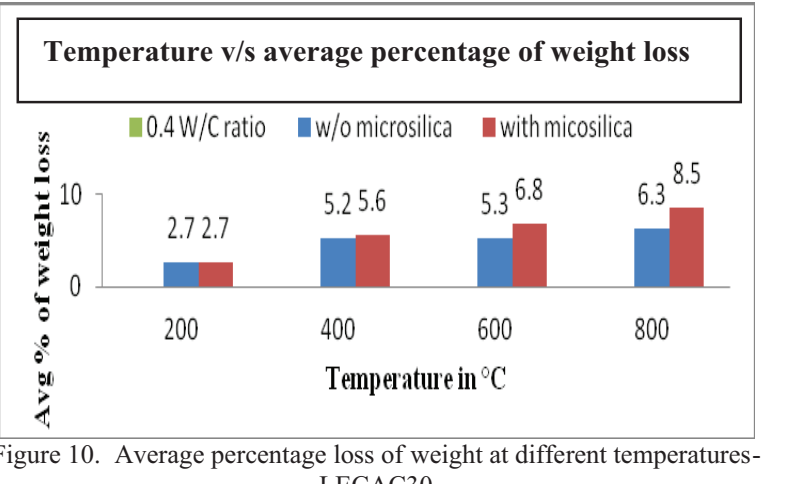
LECAC30

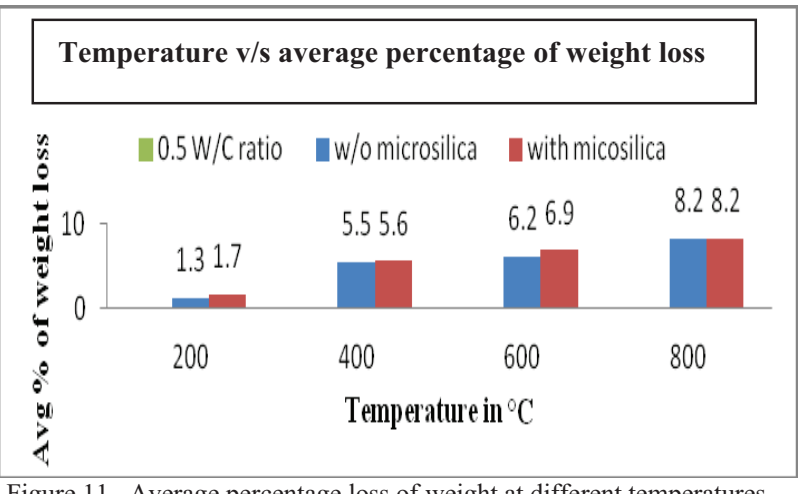

Figure 11. Average percentage loss of weight at different temperaturesLECAC30 


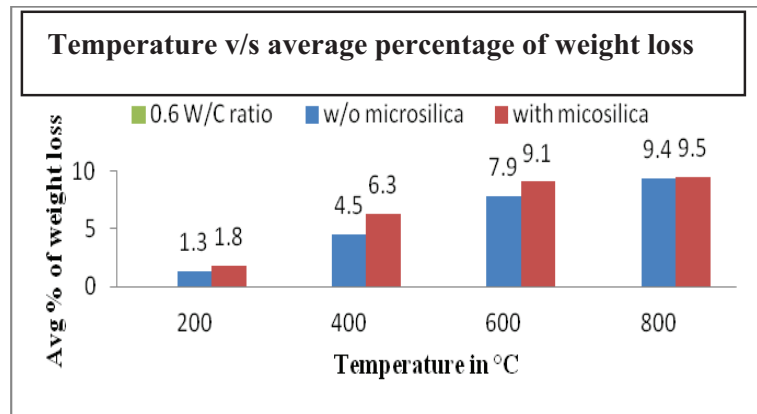

Figure 12. Average percentage loss of weight at different temperatures- LECAC30

\section{Conclusions}

The unit weight of LECAC30, LECAC40\& LECAC50 mixes was found to be in the range of $1700 \mathrm{~kg} / \mathrm{m}^{3}$ to 1890 $\mathrm{kg} / \mathrm{m}^{3}$, which is less than the unit weight of Normal Weight Aggregate concrete.

The addition of chemical admixture (Conplast SP430) improved the workability of LECAC and the optimum dosage for water- cement ratios $0.40,0.50$, and 0.60 is 1.5 $\%, 1 \%$, and $0 \%$ were respectively to achieve medium workability.

The optimum replacement of LECA to NWA for all w/b ratios $0.40,0.50$ and 0.60 is $30 \%$ i.e. $30 \%$ LECA \& $70 \%$ Gravel.

Compressive strength and flexural strength are observed to be maximum for LECAC30 at $0.4 \mathrm{w} / \mathrm{b}$ ratio with $10 \%$ Micro silica. The values obtained were $29.2 \mathrm{MPa}$ and 3.8 MPa respectively.

The addition of $10 \%$ mineral admixture (Micro silica) to NWAC, LECAC30, LECAC40 and LECAC50 results an improvement in compressive strength of $8.26 \%, 9.03 \%$, $9.86 \%$ and $19.99 \%$ respectively.

The addition of Micro silica improved the compressive strength more in LECAC50 when compared to NWAC, LECAC30 and LECAC40 as Micro silica reacts with the water available in the pores of the partially saturated surface dry LECA.

The compressive strength loss was minimum for LECAC30 with $0.4 \mathrm{w} / \mathrm{b}$ ratio at all temperatures $200^{\circ} \mathrm{C}$, $400^{\circ} \mathrm{C}, 600^{\circ} \mathrm{C}$ and $800^{\circ} \mathrm{C}$. And the average loss of compressive strength at $800^{\circ} \mathrm{C}$ temperature for NWAC and LECAC30 obtained are $74.66 \%$ and $51 \%$ respectively.

The weight loss is also minimum for LECAC30 with 0.4 $\mathrm{w} / \mathrm{b}$ ratio at all temperatures $200^{\circ} \mathrm{C}, 400^{\circ} \mathrm{C}, 600^{\circ} \mathrm{C}$ and $800^{\circ} \mathrm{C}$. And the average loss of weight at $800^{\circ} \mathrm{C}$ temperature for NWAC and LECAC30 obtained were $8.9 \%$ and $8.4 \%$ respectively.

LECAC30 has high resistance to the elevated temperatures with respect to compressive strength and weight loss.

\section{REFERENCES}

[1] Alaa M. Rashad, " Light weight expanded clay aggregate as a building material - an overview", Construction and building materials, 170, pp. 757- 775, 2018

[2] H. Costa, R. N . F. Carmo, E.Julio, "Influence of light weight aggregates concrete on the bond strength of concrete - to concrete interfaces". Construction and building materials, 180, pp. 519- 530, 2018
[3] K. Akcaozoghu, S. Akcaozoghu, "The effect of elevated temperature on the light weight concrete produced by expanded clay aggregate and calcium aluminate cement", Bilge international journal of science and technology research, 1(2): pp. $59-70,2017$.

[4] R.N.Rajprakash, A.krishnamoorthi, "Experimental study on light weight concrete using LECA", International journal of chemistry technology research, vol.10, No.8, pp 98- 109, 2017.

[5] N. Sellakkannu, C. Tamilarasan, "Feasibility study on light weight aggregates in concrete - A review", International journal for specific research and development (IJSRD) vol. 3, Issue 12, 2016.

[6] Abhijitsinh parmar, Urvish patel, "Fresh concrete properties of light weight concrete using EPS and LECA as a replacement of normal aggregates", IJEDR, vol. 4 , Issue 1, 2016.

[7] Hanmanth Shebannavar, Maneeth P. D, Brijbhushan S, "Comparative study of LECA as a replacement of coarse aggregate by ACI method with equivalent lankness of strength of IS method", International research journal of engineering and technology ( IRJET), vol. 02 Issue : 08, 2015.

[8] Anil kumar R, Dr.P. Prakash , "Mechanical properties structural light weight concrete by bleeding cinder \& LECA", International advanced research jounal in science, engineering and technology, vol . 2, Isssue 10, 2015.

[9] T. Sonia, R. Subashini, "Experimental investigation on mechanical properties of light weight concrete using LECA", (2015). International journal of science and research (IJSR), Index Copernicus value: 78.96, Impact factor : 6.391, 2015.

[10] Omar A. Abdulkareem, A.M. Mustafa Al Bakri, "Effects of elevated temperatures on the thermal behavior and mechanical performance of fly ash geopolymer paste, mortar and lightweight concrete", Construction and building materials, 50, pp. $377-387,2014$.

[11] J. Alexandre Bogas, J.de Brito, J.Cabaco, "Long term behaviour of concrete produced with recycled light weight expanded clay aggregate concrete", (2014). Construction and building materials 65 , pp. $470-479,2014$.

[12] Cheer - germ go, Jun- ren tang, "Fire resistance property of reinforced lightweight aggregate concrete wall" Construction and building materials 30, pp. $725-733,2012$. 